\title{
Exosomes and other extracellular vesicles-mediated microRNA delivery for cancer therapy
}

\author{
Giuliana Di Rocco*, Silvia Baldari*, Gabriele Toietta \\ Department of Research, Advanced Diagnostic and Technological Innovation, Translational Research Area, Regina Elena National Cancer Institute, \\ Rome, Italy \\ Contributions: (I) Conception and design: G Toietta; (II) Administrative support: None; (III) Provision of study materials or patients: None; (IV) \\ Collection and assembly of data: S Baldari, G Toietta; (V) Data analysis and interpretation: All authors; (VI) Manuscript writing: All authors; (VII) \\ Final approval of manuscript: All authors. \\ *These authors contributed equally to this work. \\ Correspondence to: Gabriele Toietta. Regina Elena National Cancer Institute, Department of Research, Advanced Diagnostic and Technological \\ Innovation, Translational Research Area, via E. Chianesi, 53, 00144 Rome, Italy. Email: gabriele.toietta@ifo.gov.it.
}

\begin{abstract}
Extracellular vesicles (EVs), including exosomes and microvesicles, are critical mediators of cellto-cell communication in tissue homeostasis and repair, both in physiological and pathological conditions. Recently, progress has been achieved in their use in regenerative medicine as transfer agents for active biomolecules. Specifically, EVs are natural carriers of microRNAs (miRNAs), protecting their cargo from plasma ribonucleases and delivering their content to recipient cells. Expression of miRNAs is dysregulated in virtually all forms of cancer. Therefore, EVs-mediated miRNA delivery may represent a valuable tool for cancer therapeutic intervention, aiming at restoring cancer miRNAs expression to normal levels. MicroRNAs may act both as tumor suppressors or oncogenes, consequently different alternative approaches for regulating miRNA expression in tumor tissues have been developed. Here, we review the various strategies for miRNA loading into EVs and highlight studies of EVs-mediated miRNA delivery which have been employed for cancer treatment, both in vitro and in vivo. Collectively, these data support the use of EVs in miRNAs/miRNAs antagonist transfer for cancer therapy, but challenges related to EV biology have yet to be further addressed before safe clinical translation.
\end{abstract}

Keywords: Exosomes; extracellular vesicles (EVs); cancer; cell-derived microparticles; microRNA; gene therapy

Submitted Jun 18, 2017. Accepted for publication Sep 10, 2017.

doi: $10.21037 /$ tcr.2017.09.29

View this article at: http://dx.doi.org/10.21037/tcr.2017.09.29

\section{Introduction}

Extracellular vesicles (EVs)

EVs are membranous vesicles originating from most cells via multivesicular bodies (exosomes), shedding from the cell membrane (microparticles), or produced by apoptotic cells (apoptotic bodies) (1) and released in many body fluids. The nature of the vesicles present in the extracellular environment is quite heterogeneous and consequently several terms have been applied to classify them (2). Discussions regarding the standardization of the isolation procedures and the identification of specific markers have been ongoing for years, but no consensus has been reached on the methods that could be implemented to unambiguously discriminate diverse classes of EVs $(3,4)$. Therefore, in agreement with a previous report (5), we prefer to use the generic term "extracellular vesicles" to collectively denote all the vesicles obtained from biological samples or cell culture supernatants, regardless of the differences in biogenesis and composition.

EVs function as intercellular messengers transferring proteins, RNA species (messenger RNA, long noncoding RNA, microRNA), DNAs (mitochondrial DNA, chromosomal DNA), carbohydrates, and bioactive lipids. 
The content of the cargo packaged into EVs differs from that in the originating cells, indicating that the loading process is selective (6). Circulating EVs can, therefore, represent powerful, minimally invasive, specific diagnostic and prognostic biomarkers for numerous diseases, including cancer $(7,8)$. On the other hand, delivery of therapeutic bioactive molecules, in particular miRNAs, through EVs may be an innovative avenue for cancer therapy (9-12). In facts, as delivery vehicles, EVs support the release of microRNAs, anti-miRNA oligonucleotides and small interfering RNAs, protecting them from degradation. Due to lack of class I/II MHC molecules expression, EVs isolated from mesenchymal cells do not promote immune response, thus allowing harvesting also from non-autologous cells and repeated administration (13). Moreover, EVs have negligible toxicity and can deliver their cargo across the blood-brain barrier (14).

\section{Micro RNAs}

Micro RNAs (miRNAs) are a class of approximately 22 nucleotide-long, non-coding RNAs predominantly involved in the regulation of target gene expression, mainly at post transcriptional level, by binding to a complementary mRNA sequences (15). More than 2,500 miRNAs have been identified in eukaryotic cells, and they have been demonstrated to play a pivotal role in regulating diverse physiological and pathological processes including cancer development, metastasis and drug resistance $(16,17)$.

Extracellular miRNAs have been identified in several biological fluids (18), protected from RNase degradation by association with Argonaute (Ago) proteins (19), in high- and low-density lipoprotein particles (20) or by inclusion into extracellular microvesicles (10). Up to $99 \%$ of circulating plasmatic miRNAs are bound to an Argonaute protein and are not enclosed within EVs (21). Argonaute are highly conserved and ubiquitously expressed proteins which play a key role in gene silencing. Ago proteins bind small non coding RNAs, including miRNAs, and mediate repression of specific target genes promoting mRNA degradation or inhibiting translation (22). Circulating miRNAs are mainly produced as "trash" RNAs from cells undergoing damage, necrosis and apoptosis. The functional role of this heterogeneous population of extracellular miRNAs is still a matter of debate $(23,24)$. Yet, several lines of evidence strongly suggest that functional miRNAs contained into EVs can be delivered to, and specifically regulate gene expression on distal target cells during viral infection, immune response and tumor progression $(25,26)$.

The ability of EVs to naturally and effectively transfer genetic information has opened up the perspective exploitation of EVs-mediated miRNAs delivery to modulate target genes expression for therapeutic purposes (27). EVs are less immunogenic and toxic in comparison to other gene therapy vectors, making them an ideal tool for local and systemic delivery of small RNA-based therapeutic molecules $(11,28,29)$. To this end, different strategies have been developed for introducing exogenous genetic material, including miRNAs and anti-miRNAs, into EVs produced by non-tumoral cells (30).

\section{Strategies for RNA loading into EVs}

Less than $10 \%$ of the RNAs present into EVs corresponds to the transcripts in the originating parental cells (27), suggesting the existence of specific cellular machineries for loading RNA into EVs $(31,32)$. The majority of the endogenous genetic material present into EVs is highly fragmented, with sequences below 700 nucleotides in length (33); a similar size limitation cutoff has been observed in studies of exogenous DNA packing into EVs (34). Therefore, EVs are unsuitable for full coding sequences transfer, but they can easily allocate and protect from endonuclease degradation non-coding miRNAs and siRNA, which are approximately 18-25 base pairs in length (26). Mechanisms of selective sorting are currently poorly characterized, but the packaging of miRNA into EVs seems to be correlated with the presence of a specific GGAG sequence, named EXOmotif (35,36).

EVs can be purified from conditional medium collected from large-scale in vitro cultures of producing cells such as mesenchymal cells (37). The yield, purity and integrity of RNA recovered from EVs are influenced by the methods of EVs purification (38). Therefore, precise analysis and accurate quantification of RNA in EVs is a challenging task, further complicated by the lack of specific standards. Notwithstanding these limitations, it has been estimated that, on average, in a mixed population of purified EVs there is less than one molecule of a given miRNA per EV (39), suggesting that either only a yet to be identified subpopulation of vesicles contains significant amounts of miRNAs or very few miRNA molecules are present in a single EV (40). At any rate, this level seems inadequate for effective target gene modulation (23). On the other hand, a single cell may produce up to $30,000 \mathrm{EVs}$ per day and up to 500 copies of miRNAs can be loaded into a single vesicle (41). 
Table 1 Methods for RNA loading into EVs

\begin{tabular}{lc}
\hline Methods & Ref. \\
\hline Pre loading strategy & \\
Transfection of miRNA mimics & $(45-49)$ \\
Transfection of plasmidic vector expressing & $(50)$ \\
specific miRNAs & \\
Electroporation of plasmidic vector expressing & \\
specific miRNAs & Baldari (u.r.) \\
Viral mediated transfer of miRNA coding \\
sequence with EXOmotif \\
Post loading strategy \\
Transfection plus heat shock of miRNA mimics \\
Electroporation of miRNA mimics \\
Virus modified EVs
\end{tabular}

u.r., unpublished results; EV, extracellular vesicle.

Therefore, by re-engineering naturally-derived EVs to increase specific miRNA payload, it might be feasible to obtain a suitable amount of EVs for clinical anti-tumor therapies (42-44). Two main approaches for loading miRNAs into EVs have been developed (Table 1) (57): the first, referred as preloading or endogenous method, involves the genetic modification of the EVs-producing cells; in the second, named post-loading or exogenous method, miRNAs are loaded into previously purified EVs (58). Each loading strategy has its advantages and limitations; the type of cells used as EVs source and/or the nature of the genetic material to deliver ultimately dictates the method of choice. For instance, by the pre-loading approach, using stable transfection or viral-mediated gene transfer, it is possible to establish genetically modified cell lines as convenient and dependable source for the in vitro production of EVs. On the other hand, transfection of primary cells with high efficiency and low toxicity, either with plasmid DNA, synthetic oligonucleotides or viral-mediated gene transfer, may represent a challenging task making the post-loading approach more suitable.

\section{Pre-loading approach}

The preloading approach is based on the observation that cellular overexpression of a selected miRNA determines the increment of its content into EVs (59). EV-producing cells can be either modified through genetic engineering using miRNA expressing plasmid or viral vectors or by introducing exogenous synthetic oligonucleotides that mimic the function of natural miRNAs, which are subsequently incorporated into secreted EVs. Possible interference of transfection reagents with $\mathrm{EV}$ s packaging should be taken into account (60). Our understanding of the cellular mechanisms of miRNAs sorting into EVs is incomplete; therefore, it is not clear whether some families of miRNAs might be preferentially packaged into EVs, making this method suitable only for some classes of miRNAs $(61,62)$. Moreover, it should be pointed out that in the cell culture supernatant the majority of miRNAs is not associated with EVs (23); consequently, the efficiency of miRNA loading into EVs is generally limited. In addition, the pre-loading method requires extensive optimization in each distinct cell type used as EVs source.

\section{Post-loading approach}

In the post-loading approach synthetic miRNA oligonucleotides are introduced by electroporation or transfection into previously isolated EVs. Potentially, this should be a more controlled process compared to the preloading approach, but increased problems related to EVs and miRNA integrity and functionality may arise (63). RNA precipitation and aggregation in the electroporation buffer have been observed during the process of transfer of siRNA oligonucleotides into EVs, making difficult to evaluate the loading efficiency, estimated to be below $0.05 \%$ (64). Refinement of the experimental condition permitted to achieve a $55 \%$ efficiency of miRNA loading into EVs (53). In contrast, other researchers have reported inefficient $\mathrm{EVs}$ loading of miRNA by electroporation and have opted for techniques based on transfection (65). Recently, a calcium phosphate co-precipitation transfection plus heat shock method for introducing miRNAs into isolated $\mathrm{EV}$ s has been reported (52). Then again, caution should be exercised in determining the loading efficiency because of the possible presence of complexes between transfection reagents and miRNAs not enclosed into EVs. The discrepancy in the success rate of miRNA loading reported by different groups may be further attributed to differences in EVs producing cells and/or in EVs purification methods.

\section{EVs for therapeutic microRNA delivery}

The use of EVs-mediated miRNA delivery to modulate target genes expression is currently under investigation as a beneficial tool for cancer therapeutics (66-68). Expression 


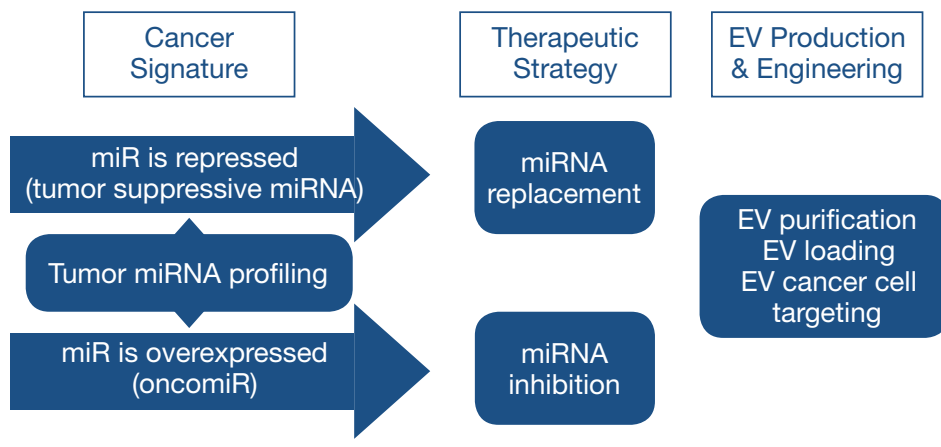

EV-mediated

miRNA delivery

Figure 1 Development of extracellular vesicles-mediated miRNA delivery strategies for cancer therapy.

of miRNAs is aberrant in practically all forms of cancer (17); therefore, reversion to normal levels is a potential way for therapeutic intervention (69). Targeting specific molecular pathways, microRNAs may act both as tumor suppressors or oncogenes (70,71); consequently, two alternative strategies are intended to reestablish physiological miRNA expression in tumor tissues, either by restoring or repressing miRNA activity (Figure 1) (72).

\section{miRNA as therapeutic agents: $E V s$-mediated miRNA replacement therapy}

Some miRNAs act as tumor suppressors promoting cancer development through down regulation of cellular oncogenes. The expression of tumor-suppressor miRNAs is lower in cancer compared to normal adjacent tissues. Therefore, restoring normal levels by miRNA replacement strategy may provide therapeutic benefit (73). Exogenous administered miRNAs are expected to function as the endogenous counterparts, minimizing the risk of off-target effects. However, it cannot be ruled out that reaching supra physiological levels, exogenously administered miRNAs may target also previously unidentified genes (11). In addition, exogenous administration of miRNAs may lead to the saturation of endogenous miRNA processing enzymes, possibly leading to perturbation of miRNAs function $(72,74)$. Furthermore, circulating miRNAs, in addition to their canonical role as post-transcriptional modulators, may act as ligands of toll like receptors, resulting in pro-tumoral stimulation (75).

\section{miRNA as therapeutic target: $E V$-mediated miRNA inbibition therapy}

The miRNAs overexpressed in cancer are considered as oncogenes and are consequently denoted as oncomiRs (71). They promote tumor development by down regulating tumor suppressive genes or genes implicated in cell differentiation or apoptosis. Therefore, the miRNA inhibition therapeutic strategy aims at inhibiting oncomiRs' expression by delivery of specific miRNA antagonists, such as antimiRNAs, locked-nucleic acids (LNA), or antagomiRNAs $(76,77)$.

\section{Preclinical studies}

Growing preclinical data support innovative EVs-based approaches for cancer therapy $(1,42)$. Here we review the most recent preclinical studies which employed miRNAs or anti-miRNAs enclosed into EVs as active agents against various types of cancers (Table 2).

EVs naturally released by adult liver stem cells may inhibit the growth of hepatoma cells in vitro and in vivo via miRNAs transfer (80). In addition, several in vitro studies have clearly demonstrated that miRNA loading into EVs, and subsequent EVs-mediated miRNAs transfer can modulate gene expression in distinctive tumor target cells including breast cancer cells (45), and osteosarcoma cells (46). Unpublished results obtained by our group suggest that lentiviral vector-mediated transfer of EXOmotif-containing miRNAs into adipose tissue-derived stromal cells may represent a suitable strategy to obtain stable production of EVs for miRNA replacement therapy. Additional studies have provided the proof-of-principle of an effective reduction of tumor growth upon treatment with miRNAloaded EV in different animal models. For instance, EVs harvested from cell culture supernatants of adipose tissuederived stromal cells transfected with a plasmid expressing miRNA-122 were able to increase hepatocellular carcinoma cells sensitivity to Sorafenib both in vitro and in a xenograft 
Table $2 \mathrm{EV}$-mediated miRNAs or anti-miRNA delivery for cancer therapy

\begin{tabular}{llll}
\hline Cargo & EV source & EV isolation & Tumor target \\
\hline MiRNA delivery & AT-MSCs & ExoQuick-TC & Hepatocellular carcinoma \\
MiR-122 & Hs578Ts cells & ExoQuick & Breast cancer \\
MiR-134 & BM MSCs & Differential centrifugation & Osteosarcoma \\
MiR-143 & BM MSCs & ExoQuick-TC & Glioma \\
MiR-146b & HEK293 cells & Differential centrifugation & Breast cancer \\
Let-7a & Dendritic cells & Differential centrifugation & Breast cancer \\
Let-7a & Macrophages & - & Colon cancer \\
MiR-143 & AT-MSC & ExoQuick TC & Hepatocellular carcinoma \\
MiR-125b & & &
\end{tabular}

ExoQuick TC, exosome precipitation reagent (System Biosciences, Mountain View, CA, USA); total exosome isolation Kit (Invitrogen, Carlsbad, CA, USA). u.r., unpublished results; EV, extracellular vesicle.

Table 3 Clinical trials investigating extracellular vesicles delivery in cancer therapy

\begin{tabular}{|c|c|c|c|}
\hline Indication & EVs source & Phase & Ref. \\
\hline Colon cancer & Ascites & Phase I & $(87)$ \\
\hline NSCLC & Dendritic cells pulsed with antigen peptides & Phase I & $(88)$ \\
\hline NSCLC & Dendritic cells & Phase I-II & NCT01159288* $(89)$ \\
\hline Colon cancer & Plant exosomes conjugated with curcumin & Phase I & NCT01294072* \\
\hline
\end{tabular}

", ClinicalTrials.gov Identifier. NSCLC, non-small cell lung cancer.

model (50). Similarly, EVs isolated from bone marrowderived stromal cells overexpressing miR-146, delivered via intra-tumor injection, reduced primary brain tumor growth in a rat xenograft model (51). Modifications of EVs to confer tumor targeting ability have been evaluated, allowing for specific therapeutic delivery of microRNA to breast cancer cells $(55,65)$.

Also EV-mediated miRNA inhibition therapy has been proven effective in experimental models of glioblastoma multiforme (78) and sarcoma (79), specifically downregulating the expression of miR-9 and miR-150, respectively.

\section{Current challenges to the clinical translation}

EVs have been recognized as paracrine mediators of cellbased therapy, generating interest on their use in clinical applications for regenerative medicine purposes (81-83). In recent years, the potential of $\mathrm{EVs}$ as vehicles for the therapy of cancer has also been proposed $(84,85)$, but published results of trials using EVs as miRNA carriers are not available yet (Table 3).

In particular, clinical trials investigating EVs delivery for cancer treatment has mainly focused on the use EVs derived from dendritic cells for tumor immunotherapy $(86,88)$. Additional studies have investigated EVs for delivery 
of chemotherapeutic drugs (Table 3). Collectively, these phase I and II trials provided evidences on the feasibility of producing clinical grade EVs and on the safety of their administration with no adverse effects observed (90).

Several clinical trials using microRNAs as therapeutic agents are currently under investigation $(91,92)$; successful clinical application requires the development of an effective and safe delivery system (29). EVs are naturallyadapted transporters of miRNAs with negligible toxicity, low immunogenicity, high stability, and which are also amenable to modifications aimed at conferring tropism as well as at improving the loading of specific cargoes $(84,93)$. Nonetheless, several issues need to be addressed before clinical use of EVs as therapeutic tools (58). Crucial problems are: (I) identification of the most proficient EVs cellular source suitable for clinical application; (II) optimization of the methods for obtaining high yields of pure EVs; (III) definition of the requirements for the characterization of purified EVs; (IV) setting of a regulatory framework for using EVs for therapeutics as advanced therapy medicinal products (58). Additional specific problems arise for the clinical translation of EVsmediated miRNA delivery for cancer therapy (85). In particular, methods for miRNA/antagomiRNA loading into EVs (94) and for precisely defining the yield of the loaded cargo should be optimized (38). A better understanding of the molecular mechanisms by which miRNAs are sorted into EVs and subsequently released by different cell types is needed (95). Purified EVs loaded with miRNAs/antimiRNAs can be delivered either systemically or through local injection into the tumor (72). For therapeutic intervention on easily accessible primary tumors such as melanoma or breast cancers, EVs can be delivered by local administration, reducing the risks of dissemination, offtarget effects and toxicity. However, for other tumors and for metastatic cancers, EVs should be delivered systemically. Though, preclinical studies suggest that exogenously EVs administered by systemic administration are rapidly cleared by the macrophages of the mononuclear phagocyte system $(81,96)$. A deeper understanding in biodistribution and pharmacokinetics profiles of EVs administered by different routes and modalities (acute $v s$. repeated administration) is required before clinical translation $(56,81)$. Interestingly, some preclinical studies have provided evidence that EVs can be engineered to enhance their targeting capability to tumor tissues (65). Cellular uptake of EVs is likely to be cell type-specific, however the mechanisms involved in the process are not completely elucidated (97). In addition, dose escalation studies of exogenously administered EVs loaded with miRNAs should be performed in order to define the therapeutic window and the maximal dose permitted without saturating the endogenous miRNA processing machinery in non tumoral cells (72).

\section{Conclusions}

EVs represent a safe vehicle for efficient delivery of therapeutic miRNAs. Strategies to further promote the therapeutic efficacy of EVs, such as methods for loading specific miRNAs/anti-miRNAs and to provide for targeted uptake by tumor cells have been proven effective in preclinical studies. Processes of pharmaceutical manufacturing of EVs suitable for clinical application are currently under investigation (98). However, before clinical application of EVs mediated miRNA delivery as a therapeutic strategy in cancer, a better understanding of EV biogenesis and functions is needed.

\section{Acknowledgements}

Funding: This work was supported by the Ministero della Salute-Ricerca Finalizzata (RF-2011-02347907 to G.T.).

\section{Footnote}

Conflicts of Interest: The authors have no conflicts of interest to disclose.

\section{References}

1. Kalra H, Drummen GP, Mathivanan S. Focus on extracellular vesicles: introducing the next small big thing. Int J Mol Sci 2016;17:170.

2. van der Pol E, Böing AN, Gool EL, et al. Recent developments in the nomenclature, presence, isolation, detection and clinical impact of extracellular vesicles. J Thromb Haemost 2016;14:48-56.

3. Lötvall J, Hill AF, Hochberg F, et al. Minimal experimental requirements for definition of extracellular vesicles and their functions: a position statement from the International Society for Extracellular Vesicles. J Extracell Vesicles 2014;3:26913.

4. Witwer KW, Buzás EI, Bemis LT, et al. Standardization of sample collection, isolation and analysis methods in extracellular vesicle research. J Extracell Vesicles $2013 ; 20360$. 
5. Gould SJ, Raposo G. As we wait: coping with an imperfect nomenclature for extracellular vesicles. J Extracell Vesicles 2013;20389.

6. Villarroya-Beltri C, Baixauli F, Gutiérrez-Vázquez C, et al. Sorting it out: regulation of exosome loading. Semin Cancer Biol 2014;28:3-13.

7. Munson P, Shukla A. Exosomes: Potential in Cancer Diagnosis and Therapy. Medicines (Basel) 2015;2:310-27.

8. Guo L, He B. Extracellular vesicles and their diagnostic and prognostic potential in cancer. Transl Cancer Res 2017;6:599-612.

9. Shah MY, Ferrajoli A, Sood AK, et al. microRNA therapeutics in cancer - An emerging concept. EBioMedicine 2016;12:34-42.

10. Yu X, Odenthal M, Fries JW. Exosomes as miRNA carriers: Formation-function-future. Int J Mol Sci 2016;17. pii: E2028.

11. Rupaimoole R, Slack FJ. MicroRNA therapeutics: towards a new era for the management of cancer and other diseases. Nat Rev Drug Discov 2017;16:203-22.

12. Bastos N, Ruivo CF, da Silva S, et al. Exosomes in cancer: Use them or target them? Semin Cell Dev Biol 2017. [Epub ahead of print].

13. Muraca M, Piccoli M, Franzin C, et al. Diverging concepts and novel perspectives in regenerative medicine. Int $\mathrm{J} \mathrm{Mol}$ Sci 2017;18. pii: E1021.

14. Shahabipour F, Barati N, Johnston TP, et al. Exosomes: Nanoparticulate tools for RNA interference and drug delivery. J Cell Physiol 2017;232:1660-8.

15. Hammond SM. An overview of microRNAs. Adv Drug Deliv Rev 2015;87:3-14.

16. Croce CM. Causes and consequences of microRNA dysregulation in cancer. Nat Rev Genet 2009;10:704-14.

17. Peng Y, Croce CM. The role of MicroRNAs in human cancer. Signal Transduction and Targeted Therapy 2016;1:15004.

18. Makarova JA, Shkurnikov MU, Wicklein D, et al. Intracellular and extracellular microRNA: An update on localization and biological role. Prog Histochem Cytochem 2016;51:33-49.

19. Daugaard I, Hansen TB. Biogenesis and function of AgoAssociated RNAs. Trends Genet 2017;33:208-19.

20. Vickers KC, Palmisano BT, Shoucri BM, et al. MicroRNAs are transported in plasma and delivered to recipient cells by high-density lipoproteins. Nat Cell Biol 2011;13:423-33.

21. Turchinovich A, Weiz L, Langheinz A, et al. Characterization of extracellular circulating microRNA.
Nucleic Acids Res 2011;39:7223-33.

22. Meister G. Argonaute proteins: functional insights and emerging roles. Nat Rev Genet 2013;14:447-59.

23. Turchinovich A, Weiz L, Burwinkel B. Extracellular miRNAs: the mystery of their origin and function. Trends Biochem Sci 2012;37:460-5.

24. Turchinovich A, Tonevitsky AG, Burwinkel B. Extracellular miRNA: A collision of two paradigms. Trends Biochem Sci 2016;41:883-92.

25. Chen X, Liang H, Zhang J, et al. Secreted microRNAs: a new form of intercellular communication. Trends Cell Biol 2012;22:125-32.

26. Zomer A, Vendrig T, Hopmans ES, et al. Exosomes: Fit to deliver small RNA. Commun Integr Biol 2010;3:447-50.

27. Lee Y, El Andaloussi S, Wood MJ. Exosomes and microvesicles: extracellular vesicles for genetic information transfer and gene therapy. Hum Mol Genet 2012;21:R125-34.

28. Tan A, Rajadas J, Seifalian AM. Exosomes as nanotheranostic delivery platforms for gene therapy. Adv Drug Deliv Rev 2013;65:357-67.

29. Tyagi N, Arora S, Deshmukh SK, et al. Exploiting nanotechnology for the development of microRNAbased cancer therapeutics. J Biomed Nanotechnol 2016;12:28-42.

30. Jiang L, Vader P, Schiffelers RM. Extracellular vesicles for nucleic acid delivery: progress and prospects for safe RNAbased gene therapy. Gene Ther 2017;24:157-66.

31. Zhang J, Li S, Li L, et al. Exosome and exosomal microRNA: trafficking, sorting, and function. Genomics Proteomics Bioinformatics 2015;13:17-24.

32. Abels ER, Breakefield XO. Introduction to extracellular vesicles: biogenesis, RNA cargo selection, content, release, and uptake. Cell Mol Neurobiol 2016;36:301-12.

33. Batagov AO, Kurochkin IV. Exosomes secreted by human cells transport largely mRNA fragments that are enriched in the 3'-untranslated regions. Biol Direct 2013;8:12.

34. Lamichhane TN, Raiker RS, Jay SM. Exogenous DNA loading into extracellular vesicles via electroporation is size-dependent and enables limited gene delivery. Mol Pharm 2015;12:3650-7.

35. Villarroya-Beltri C, Gutiérrez-Vázquez C, Sánchez-Cabo F, et al. Sumoylated hnRNPA2B1 controls the sorting of miRNAs into exosomes through binding to specific motifs. Nat Commun 2013;4:2980.

36. Kim KM, Abdelmohsen K, Mustapic M, et al. RNA in extracellular vesicles. Wiley Interdiscip Rev RNA 2017;8:e1413. 
37. Pachler K, Lener T, Streif D, et al. A Good Manufacturing Practice-grade standard protocol for exclusively human mesenchymal stromal cell-derived extracellular vesicles. Cytotherapy 2017;19:458-72.

38. Mateescu B, Kowal EJ, van Balkom BW, et al. Obstacles and opportunities in the functional analysis of extracellular vesicle RNA - an ISEV position paper. J Extracell Vesicles 2017;6:1286095.

39. Chevillet JR, Kang Q, Ruf IK, et al. Quantitative and stoichiometric analysis of the microRNA content of exosomes. Proc Natl Acad Sci U S A 2014;111:14888-93.

40. Tkach M, Théry C. Communication by extracellular vesicles: where we are and where we need to go. Cell 2016;164:1226-32.

41. Koppers-Lalic D, Hogenboom MM, Middeldorp JM, et al. Virus-modified exosomes for targeted RNA delivery; a new approach in nanomedicine. Adv Drug Deliv Rev 2013;65:348-56.

42. György B, Hung ME, Breakefield XO, et al. Therapeutic applications of extracellular vesicles: clinical promise and open questions. Annu Rev Pharmacol Toxicol 2015;5 5:439-64.

43. Watson DC, Bayik D, Srivatsan A, et al. Efficient production and enhanced tumor delivery of engineered extracellular vesicles. Biomaterials 2016;105:195-205.

44. Gilligan KE, Dwyer RM. Engineering exosomes for cancer therapy. Int J Mol Sci 2017;18:1122.

45. O'Brien K, Lowry MC, Corcoran C, et al. miR-134 in extracellular vesicles reduces triple-negative breast cancer aggression and increases drug sensitivity. Oncotarget 2015;6:32774-89.

46. Shimbo K, Miyaki S, Ishitobi H, et al. Exosome-formed synthetic microRNA-143 is transferred to osteosarcoma cells and inhibits their migration. Biochem Biophys Res Commun 2014;445:381-7.

47. Akao Y, Iio A, Itoh T, et al. Microvesicle-mediated RNA molecule delivery system using monocytes/macrophages. Mol Ther 2011;19:395-9.

48. Lee ST, Im W, Ban JJ, et al. Exosome-based delivery of miR-124 in a Huntington's disease model. J Mov Disord 2017;10:45-52.

49. Rechavi O, Erlich Y, Amram H, et al. Cell contactdependent acquisition of cellular and viral nonautonomously encoded small RNAs. Genes Dev 2009;23:1971-9.

50. Lou G, Song X, Yang F, et al. Exosomes derived from miR-122-modified adipose tissue-derived MSCs increase chemosensitivity of hepatocellular carcinoma. J Hematol
Oncol 2015;8:122.

51. Katakowski M, Buller B, Zheng X, et al. Exosomes from marrow stromal cells expressing miR-146b inhibit glioma growth. Cancer Lett 2013;335:201-4.

52. Zhang D, Lee H, Zhu Z, et al. Enrichment of selective miRNAs in exosomes and delivery of exosomal miRNAs in vitro and in vivo. Am J Physiol Lung Cell Mol Physiol 2017;312:L110-L21.

53. Momen-Heravi F, Bala S, Bukong T, et al. Exosomemediated delivery of functionally active miRNA-155 inhibitor to macrophages. Nanomedicine 2014;10:1517-27.

54. Yang Z, Xie J, Zhu J, et al. Functional exosome-mimic for delivery of siRNA to cancer: in vitro and in vivo evaluation. J Control Release 2016;243:160-71.

55. Wang Y, Chen X, Tian B, et al. Nucleolin-targeted extracellular vesicles as a versatile platform for biologics delivery to breast cancer. Theranostics 2017;7:1360-72.

56. Bala S, Csak T, Momen-Heravi F, et al. Biodistribution and function of extracellular miRNA-155 in mice. Sci Rep 2015;5:10721.

57. Janas T, Janas MM, Sapoń K. Mechanisms of RNA loading into exosomes. FEBS Lett 2015;589:1391-8.

58. Lener T, Gimona M, Aigner L, et al. Applying extracellular vesicles based therapeutics in clinical trials an ISEV position paper. J Extracell Vesicles 2015;4:30087.

59. Squadrito ML, Baer C, Burdet F, et al. Endogenous RNAs modulate microRNA sorting to exosomes and transfer to acceptor cells. Cell Rep 2014;8:1432-46.

60. Zhou Y, Zhou G, Tian C, et al. Exosome-mediated small RNA delivery for gene therapy. Wiley Interdiscip Rev RNA 2016;7:758-71.

61. Cha DJ, Franklin JL, Dou Y, et al. KRAS-dependent sorting of miRNA to exosomes. Elife 2015;4:e07197.

62. Batagov AO, Kuznetsov VA, Kurochkin IV. Identification of nucleotide patterns enriched in secreted RNAs as putative cis-acting elements targeting them to exosome nano-vesicles. BMC Genomics 2011;12 Suppl 3:S18.

63. Vader P, Kooijmans SA, Stremersch S, et al. New considerations in the preparation of nucleic acid-loaded extracellular vesicles. Ther Deliv 2014;5:105-7.

64. Kooijmans SA, Stremersch S, Braeckmans K, et al. Electroporation-induced siRNA precipitation obscures the efficiency of siRNA loading into extracellular vesicles. J Control Release 2013;172:229-38.

65. Ohno S, Takanashi M, Sudo K, et al. Systemically injected exosomes targeted to EGFR deliver antitumor microRNA to breast cancer cells. Mol Ther 2013;21:185-91.

66. Kosaka N, Takeshita F, Yoshioka Y, et al. Exosomal tumor- 
suppressive microRNAs as novel cancer therapy: "exocure" is another choice for cancer treatment. Adv Drug Deliv Rev 2013;65:376-82.

67. Ma P, Pan Y, Li W, et al. Extracellular vesicles-mediated noncoding RNAs transfer in cancer. J Hematol Oncol 2017;10:57.

68. Ji W, Sun B, Su C. Targeting microRNAs in cancer gene therapy. Genes (Basel) 2017;8:21.

69. Pereira DM, Rodrigues PM, Borralho PM, et al. Delivering the promise of miRNA cancer therapeutics. Drug Discov Today 2013;18:282-9.

70. Zhang B, Pan X, Cobb GP, et al. microRNAs as oncogenes and tumor suppressors. Dev Biol 2007;302:1-12.

71. Svoronos AA, Engelman DM, Slack FJ. OncomiR or tumor suppressor? The duplicity of microRNAs in cancer. Cancer Res 2016;76:3666-70.

72. Chen Y, Gao DY, Huang L. In vivo delivery of miRNAs for cancer therapy: challenges and strategies. Adv Drug Deliv Rev 2015;81:128-41.

73. Bader AG, Brown D, Winkler M. The promise of microRNA replacement therapy. Cancer Res 2010;70:7027-30.

74. Khan AA, Betel D, Miller ML, et al. Transfection of small RNAs globally perturbs gene regulation by endogenous microRNAs. Nat Biotechnol 2009;27:549-55.

75. Fabbri M, Paone A, Calore F, et al. A new role for microRNAs, as ligands of Toll-like receptors. RNA Biol 2013;10:169-74.

76. Li Z, Rana TM. Therapeutic targeting of microRNAs: current status and future challenges. Nat Rev Drug Discov 2014;13:622-38.

77. Zhang Y, Wang Z, Gemeinhart RA. Progress in microRNA delivery. J Control Release 2013;172:962-74.

78. Munoz JL, Bliss SA, Greco SJ, et al. Delivery of functional anti-miR-9 by mesenchymal stem cell-derived exosomes to glioblastoma multiforme cells conferred chemosensitivity. Mol Ther Nucleic Acids 2013;2:e126.

79. Liu Y, Zhao L, Li D, et al. Microvesicle-delivery miR-150 promotes tumorigenesis by up-regulating VEGF, and the neutralization of miR-150 attenuate tumor development. Protein Cell 2013;4:932-41.

80. Fonsato V, Collino F, Herrera MB, et al. Human liver stem cell-derived microvesicles inhibit hepatoma growth in SCID mice by delivering antitumor microRNAs. Stem Cells 2012;30:1985-98.

81. Di Rocco G, Baldari S, Toietta G. Towards therapeutic delivery of extracellular vesicles: Strategies for in vivo tracking and biodistribution analysis. Stem Cells Int
2016;2016:5029619.

82. Fuster-Matanzo A, Gessler F, Leonardi T, et al. Acellular approaches for regenerative medicine: on the verge of clinical trials with extracellular membrane vesicles? Stem Cell Res Ther 2015;6:227.

83. Riazifar M, Pone EJ, Lötvall J, et al. Stem cell extracellular vesicles: Extended messages of regeneration. Annu Rev Pharmacol Toxicol 2017;57:125-54.

84. H Rashed M, Bayraktar E, K Helal G, et al. Exosomes: From garbage bins to promising therapeutic targets. Int J Mol Sci 2017;18. pii: E538.

85. Yamada NO. Extracellular vesicles in cancer: current status and challenges. Transl Cancer Res 2017;5:59.

86. Escudier B, Dorval T, Chaput N, et al. Vaccination of metastatic melanoma patients with autologous dendritic cell (DC) derived-exosomes: results of thefirst phase I clinical trial. J Transl Med 2005;3:10.

87. Dai S, Wei D, Wu Z, et al. Phase I clinical trial of autologous ascites-derived exosomes combined with GMCSF for colorectal cancer. Mol Ther 2008;16:782-90.

88. Morse MA, Garst J, Osada T, et al. A phase I study of dexosome immunotherapy in patients with advanced nonsmall cell lung cancer. J Transl Med 2005;3:9.

89. Besse B, Charrier M, Lapierre V, et al. Dendritic cellderived exosomes as maintenance immunotherapy after first line chemotherapy in NSCLC. Oncoimmunology 2015;5:e1071008.

90. Ohno S, Drummen GP, Kuroda M. Focus on extracellular vesicles: development of extracellular vesicle-based therapeutic systems. Int J Mol Sci 2016;17:172.

91. Christopher AF, Kaur RP, Kaur G, et al. MicroRNA therapeutics: Discovering novel targets and developing specific therapy. Perspect Clin Res 2016;7:68-74.

92. Wang J, Zheng Y, Zhao M. Exosome-based cancer therapy: Implication for targeting cancer stem cells. Front Pharmacol 2017;7:533.

93. Barile L, Vassalli G. Exosomes: Therapy delivery tools and biomarkers of diseases. Pharmacol Ther 2017;174:63-78.

94. Sutaria DS, Badawi M, Phelps MA, et al. Achieving the promise of therapeutic extracellular vesicles: The devil is in details of therapeutic loading. Pharm Res 2017;34:1053-66.

95. Thind A, Wilson C. Exosomal miRNAs as cancer biomarkers and therapeutic targets. J Extracell Vesicles 2016;5:31292.

96. Takahashi Y, Nishikawa M, Shinotsuka H, et al. Visualization and in vivo tracking of the exosomes of murine melanoma B16-BL6 cells in mice after intravenous 
injection. J Biotechnol 2013;165:77-84.

97. Mulcahy LA, Pink RC, Carter DR. Routes and mechanisms of extracellular vesicle uptake. J Extracell Vesicles 2014;3:24641.

Cite this article as: Di Rocco G, Baldari S, Toietta G. Exosomes and other extracellular vesicles-mediated microRNA delivery for cancer therapy. Transl Cancer Res 2017;6(Suppl 8):S1321-S1330. doi: 10.21037/tcr.2017.09.29
98. Gimona M, Pachler K, Laner-Plamberger S, et al. Manufacturing of human extracellular vesicle-based therapeutics for clinical use. Int J Mol Sci 2017;18:1190. 